\title{
HI in Local Group analogs: what does it tell us about galaxy formation?
}

\author{
D.J. Pisano ${ }^{1}$, D.G. Barnes ${ }^{2}$, B.K. Gibson ${ }^{3}$, L. Staveley-Smith ${ }^{4}$, K.C. \\ Freeman $^{5}$, V.A. Kilborn ${ }^{6}$ \\ 1 Naval Research Laboratory, 4555 Overlook Ave. SW, Washington, DC 20375 \\ USA; pisano@nrl.navy.mil \\ 2 School of Physics, University of Melbourne, Victoria 3010, Australia; \\ dbarnes@astro.ph.unimelb.edu.au \\ 3 Centre for Astrophysics, University of Central Lancashire, Preston, PR1 2HE, \\ UK; bkgibson@uclan.ac.uk \\ 4 Australia Telescope National Facility, P.O. Box 76, Epping NSW 1710, \\ Australia; Lister.Staveley-Smith@atnf.csiro.au \\ 5 RSAA, Mount Stromlo Observatory, Cotter Road, Weston, ACT 2611, \\ Australia; kcf@mso . anu .edu . au \\ 6 Centre for Astrophysics \& Supercomputing, Swinburne University, Hawthorn, \\ Victoria 3122, Australia; vkilborn@astro.swin.edu.au
}

\begin{abstract}
Summary. We present the results of our HI survey of six loose groups of galaxies analogous to the Local Group. The survey was conducted using the Parkes telescope and the Australia Telescope Compact Array to produce a census of all the gasrich galaxies and potential analogs to the high-velocity clouds (HVCs) within these groups down to $M_{H I} \leq 10^{7} \mathrm{M}_{\odot}$ as a test of models of galaxy formation. We present the HI mass function and halo mass function for these analogous groups and compare them with the Local Group and other environments. We also demonstrate that our non-detection of HVC analogs in these groups implies that they must have low HI masses and be clustered tightly around galaxies, including around our own Milky Way, and are not distributed throughout the Local Group.
\end{abstract}

\section{Introduction}

As various proceedings from this conference illustrate, the Local Group is the nearest and best studied group of galaxies. We have a census of the dwarf galaxies down to very faint magnitude limits. We can study the resolved stellar populations in Local Group galaxies using HST and large ground-based telescope and re-create their star formation histories. These same data can be used to measure distances to these galaxies. We can now even measure the proper motions of some Local Group galaxies 4. But just as our position within the Milky Way complicates our studies of it, our position inside the Local Group leads to similar problems. This is particularly true when studying 
the gas within the Local Group that is not associated with stars, for example the high-velocity clouds (HVCs) 19.

The HVCs were first discovered over 40 years ago 12 and have remained a mystery since. They are HI clouds which are moving inconsistent with simple Galactic rotation with deviation velocities greater than $90 \mathrm{~km} \mathrm{~s}^{-1}$. They also lack stars [20], and so we are generally unable to directly infer their distances. Without knowing their distances, we do not know their masses and can not, therefore, divine the physical processes responsible for their origins. Most likely, however, they represent a variety of phenomena. Some are probably related to a galactic fountain powered by supernovae explosions [3] and are located in the lower Galactic halo. Some HVCs are certainly tidal in origin, like the Magellanic Stream [14. Others may be infalling primordial gas, such as Complex C [18], or gas condensing out of a hot Galactic halo 9]. Finally, some have been suggested to be associated with the low mass dark matter halos predicted to exist by models of cold dark matter (CDM) galaxy formation 1. 2.

CDM models predict that the Local Group should contain $~ 300$ low mass dark halos, while there are only $\sim 20$ luminous dwarf galaxies known [8, 11]. While this may imply that we lack a complete census of the luminous galaxies in the Local Group, it may also be uniquely deficient in dwarf galaxies. Or, perhaps, the HVCs may populate these dark matter halos and solve this "missing satellite" problem.

The best way to address these questions is to study other groups of galaxies in detail. We can then determine if the population of low mass, gas-rich galaxies in the Local Group typical, or if there are relatively more in analogous groups? If HVCs are the solution to the "missing satellite" problem, then they should be ubiquitous in other groups where we can then directly determine their masses. Our HI survey of six loose groups of galaxies chosen to be analogous to the Local Group attempts to answer these questions.

\section{Observations}

We selected five of our groups from the optical catalog of [7] and one group from the HIPASS catalog [10]. The resulting groups are between 10.6 and 13.4 Mpc distant, contain between 3-9 bright, spiral galaxies which are separated, on average, by $\sim 550 \mathrm{kpc}$, and have diameters of $\sim 1.6 \mathrm{Mpc}$; hence the moniker loose groups. These groups were known to contain only spiral and irregular galaxies and no massive elliptical galaxies; just like the Local Group. These groups have no known X-ray emitting gas as is expected to be the case for the Local Group. Their masses, as estimated by the virial theorem, of $10^{11.7-13.6} \mathrm{M}_{\odot}$ are comparable to the mass of the Local Group $\sim 10^{12.4} \mathrm{M}_{\odot}$ [5].

We used the Parkes Multibeam and Australia Telescope Compact Array (ATCA) to survey the entire area of each group down to a $M_{H I}$ sensitivity 
of $5-8 \times 10^{5} \mathrm{M}_{\odot}$ per $3.3 \mathrm{~km} \mathrm{~s} \mathrm{~s}^{-1}$. All Parkes detections in the groups were confirmed to be real by the follow-up ATCA observations. A total of 64 HI-rich galaxies were detected in the six groups, almost twice the number of optically cataloged group galaxies [7] and $50 \%$ more galaxies than were detected by HIPASS in the same fields [10. All of our detections are associated with optical counterparts and have properties consistent with typical spiral, irregular, or dwarf irregular galaxies. No analogs to the HVCs were detected.

\section{Is the Local Group typical?}

While our sample of loose groups was chosen to be qualitatively analogous to the Local Group, are the HI properties of these groups quantitatively similar? To answer this, we constructed an HI mass function (HIMF) and a cumulative circular velocity distribution function (CVDF), both shown in Figure 1. For the former we have not made any attempt to quantify the volume observed, so we are simply comparing the slopes. That is, we are comparing the ratio of high- $M_{H I}$ and low- $M_{H I}$ galaxies in our sample to the Local Group. The CVDF is a measure of the dark matter halo mass function as traced by luminous matter, in this case only for HI-rich galaxies. As for the HIMF, we are focusing on the slope of the CVDF and how our groups compare to the Local Group and CDM model predictions.
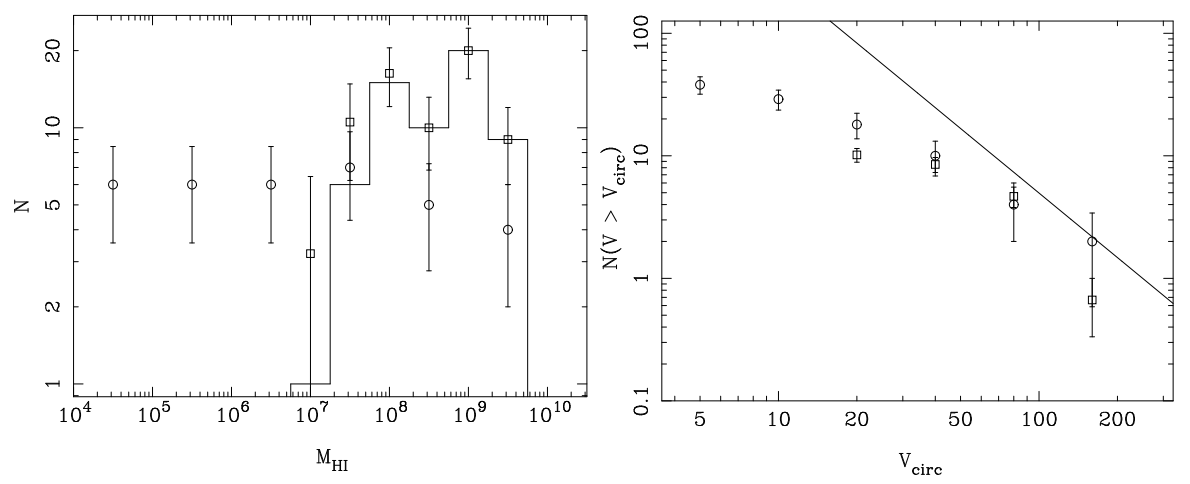

Fig. 1. Left: The HI mass function (HIMF) of the Local Group (circles) and the sum of the six loose groups (solid histogram) corrected for incompleteness (squares). Right: The cumulative velocity distribution function for the Local Group (circles), and the average of the six loose groups (squares). The solid line represents the CDM model from [8], roughly normalized to the second data point.

The HIMF for both the Local Group and our sample of loose groups appears to be flat; there are the same number of massive and low mass galaxies per dex of $M_{H I}$ when our sample is corrected for incompleteness down to the 
sensitivity limit of our survey. This is consistent with other results for the HIMF in low density environments. In 22, it was shown that the HIMF flattens in lower density environments with data from HIPASS, although others find an opposite trend [15]. In [17], similar results to [22] were demonstrated from a compilation of optical luminosity functions. This demonstrates that, as far as the $M_{H I}$ distribution goes, the Local Group is not atypical.

The CVDF for the Local Group clearly deviates from the solid line representing the predictions from CDM models [8] below $V_{\text {circ }} \sim 80 \mathrm{~km} \mathrm{~s}^{-1}$; this is the "missing-satellite" problem. From Figur_1 we can see that our loose groups show the exact same deviation and match the CVDF for the Local Group within the statistical errors. Note that this is the CVDF only for the gas-rich galaxies in these groups, and we know that there are more gas-poor systems within the Local Group. These are shown in Figure 1 but typically have lower circular velocities than the gas-rich systems. The CVDF for the loose groups has not been corrected for incompleteness, but we do not expect such corrections to be large. This comparison of CVDFs illustrates, again, that there does not appear to a deficit of dwarf galaxies in the Local Group as compared to other loose groups. The Local Group does not appear to be an atypical loose group.

\section{The nature of the High Velocity Clouds}

Since we can now be confident that our sample of loose groups is truly analogous to the Local Group, what does our non-detection of HVCs in these groups imply for their nature? To answer this question, we have constructed a simple model with a Monte Carlo simulation for the distribution of the HVCs as described in 13. For this paper, we use our knowledge of the completeness of our survey as a function of integrated flux and linewidth.

Based on this simulation, our non-detection of any HVC analogs in these six loose groups implies that they must have relatively low average HI masses, $M_{H I} \leq 1.6 \times 10^{5} \mathrm{M}_{\odot}$, and be tightly clustered around individual galaxies, $D_{H W H M} \leq 100 \mathrm{kpc}$. These constraints are consistent with the limits found by others through a variety of other observational and theoretical methods 21, 6. 16. This also implies that CHVCs are not a major repository of neutral gas, $M_{H I} \leq 10^{8} \mathrm{M}_{\odot}$, although there may be a large reservoir of ionized gas to fuel star formation in the Milky Way. These limits are independent of the nature of HVCs; they may or may not be associated with dark matter halos [9].

\section{Conclusions}

We have conducted a deep HI survey of six loose groups of galaxies analogous to the Local Group. The survey yielded detections of 64 HI-rich dwarf galaxies. The slopes of the HI mass function and the luminous, dark matter halo 
function for the Local Group and our sample of loose groups are consistent with each other. The Local Group, therefore, does not appear to be atypical in its apparent deficit of low mass, dwarf galaxies as compared to the predictions of CDM models of galaxy formation. This does not necessarily imply a failure of CDM, but does require, at least, the suppression of baryon collapse in low mass halos.

Our survey also failed to detect any HI clouds lacking stars; no HVC analogs were detected. This implies that HVCs are not a large reservoir of neutral gas for future star formation in galaxies. This is consistent with many models for the origin of HVCs and with other observational limits. Both deeper HI surveys of the $\sim 100 \mathrm{kpc}$ environment of individual galaxies and higher resolution and sensitivity observations of Milky Way HVCs will help to better constrain the nature and origin of HVCs

This research was performed while D.J.P. held a National Research Council Research Associateship Award at the Naval Research Laboratory. Basic research in astronomy at the Naval Research Laboratory is funded by the Office of Naval Research. D.J.P. also acknowledges generous support from the ATNF via a Bolton Fellowship and from NSF MPS Distinguished International Research Fellowship grant AST 0104439.

\section{References}

1. Blitz, L., Spergel, D.N., Teuben, P.J. et al: ApJ 514, 818 (1999)

2. Braun, R., Burton, W.B.: A\&A 341, 437 (1999)

3. Bregman, J.N.: ApJ 234, 577 (1980)

4. Brunthaler, A., Reid, M.J., Falcke, H., et al: Science 307, 1440 (2005)

5. Courteau, S., van den Bergh, S.: AJ 118, 337 (1999)

6. de Heij, V., Braun, R., Burton, W.B.: A\&A 392417 (2002)

7. Garcia, A.M.: A\&AS 10047 (1993)

8. Klypin, A., Kravtsov, A.V., Valenzuela, O. et al: ApJ 522, 82 (1999)

9. Maller, A.H., Bullock, J.S.: MNRAS 355, 694 (2004)

10. Meyer, M.J., Zwaan, M.A., Webster, R.L., et al: MNRAS 350, 1195 (2004)

11. Moore, B., Ghigna, S., Governato, F., et al: ApJ 524, L19 (1999)

12. Muller, C.A., Oort, J.H., Raimond, E.: CR Acad. Sci. Paris 257, 1661 (1963)

13. Pisano, D.J., Barnes, D.G., Gibson, B.K., et al: ApJ 610, L17 (2004)

14. Putman, M.E., Gibson, B.K., Staveley-Smith, L., et al: Nature 394, 752 (1998)

15. Springob, C.M., Haynes, M.P., Giovanelli, R.: ApJ 621, 215 (2005)

16. Thilker, D.A., Braun, R., Walterbos, R.A.M., et al: ApJ 601, L39 (2004)

17. Tully, R.B., Somerville, R.S., Trentham, N., et al: ApJ 569, 573 (2002)

18. Wakker, B.P., Howk, J.C., Savage, B.D., et al.: Nature 402, 388 (1999)

19. Wakker, B.P., van Woerden, H.: ARA\&A 35, 217 (1997)

20. Willman, B., Dalcanton, J., Ivezic, Z., et al: AJ 124, 2600 (2002)

21. Zwaan, M.A.: MNRAS 325, 1142 (2001)

22. Zwaan, M.A., Meyer, M.J., Staveley-Smith, L., et al: MNRAS 359, L30 (2005) 